\title{
Behavior of Position based Routing in VANET
}

\author{
Jatinder Kumar \\ Research Scholar, \\ Department of ECE \\ S.B.S. STC, Ferozepur, \\ Punjab
}

\author{
Vikram Mutneja \\ Assistant Professor, \\ Department of ECE \\ S.B.S. STC, Ferozepur, \\ Punjab
}

\author{
Inderjeet Singh Gill \\ Assistant Professor, \\ Department of ECE \\ S.B.S.STC, Ferozepur, Punjab
}

\begin{abstract}
Over the last few years a new field of technology i.e. Vehicular Ad-hoc Networks (VANET) has been generated through Wireless Sensor Networks (WSN) and it attracts very large number of researchers. Now VANET has become a very interesting and developing area in the WSN, because it provides traffic and road safety by connecting vehicles travelling on the road. Also it provides communication between moving vehicles to the other vehicles. In VANET, data is transferred from one vehicle to another, so the security is a major issue, because any successful attack in VANET can cause large destruction. Routing between the moving vehicles is very challenging and quite interesting. This paper includes the Position Based Routing (PBR) protocol to provide the connection between moving vehicles on the road for the purpose of safety, communication and also for the driver's comfort. In this we are evaluating the GPSR (Greedy Perimeter Stateless Routing) which is very popular Position Based Routing in VANET. In order to evaluate the realistic simulation environment for vehicles we are using NS2 (Network Simulator 2) and SUMO (Simulation for Urban Mobility). The performance is measured in the form of some parameters like Network throughput, Packet delivery ratio (PDR), NRL (Network Routing Load) and Average end to end delay.
\end{abstract}

\section{Keywords}

VANET, Greedy Perimeter Stateless Routing, Position Based Routing, SUMO and NS2.

\section{INTRODUCTION}

There are very large number of vehicles travel on the road, so the number of accidents happened on the road is also large. To provide the road safety and comfort for drivers, a new research area is developed by WSN i.e. Vehicular Ad-hoc Networks. Basically VANET is divided into two categories i.e. Safety and Non safety applications [1]. Driver drowsiness prevention system, Collision avoidance, Automatic emergency braking systems are included in the safety applications and on the other side the systems like Direction changer for traffic information, automatic toll service, cooperative entertainment are some of the Non safety applications.[2]

In VANET the connection established is only for few seconds, because the speed of the moving vehicles is very fast. So the topology is dynamic and not fixed in VANET [3]-[4]. In this we are evaluating the GPSR which is very popular Position Based Routing protocol. In Location based approaches with the help of on-board navigation systems, the vehicles are aware of their geographical positions [5]. Other than the destination node position, each node knows its own position and the position of its one hop neighbor in order to forward packets [6].
The major attacks in VANETs are message forging, impersonation, packet dropping, black hole, grey hole, worm hole, on-board tampering, and in-transit traffic tampering [7]. In VANET the nodes have high mobility so the change in topology is also very fast [11]-[14]. In PBR to make the routing decisions, the nodes use the geographical information [12]-[13]-[14]. In dynamic scenarios, the PBR performs better but in some highly dynamic scenarios like VANET, they don't perform efficiently [15].

This paper is organized as follows: Section 1 gives the basic information about the topic. Section 2 describes the Literature Review. Section 3 defines the various performance parameters. Section 4 describes the simulation environment in which various cases are defined in SUMO and NS2. The Section 5 is Results and Discussions in which results are shown in the form of parameters. Section 6 defines the Conclusion and Future work.

\section{LITERATURE REVIEW}

Now these days large amount of research have done in the field of WSN, but many topics are untouched in it. It is one of the very attractive and developing areas. It is also very interesting area, so that it attracts large number of researchers to explore the area.

We do literature review to ensure the thorough understanding of the topic, to identify potential areas for research and the similar work done within the area.

Yasser Toor and Paul Muhlethaler [3] did a survey and concluded that the connection established between moving vehicles is only for few seconds, because the speed of the vehicles is very fast. The topology is not fixed and dynamic in nature.

Hannes Hartenstein and Dieter Vollmer [5] did a survey on Location based routing for VANETs and evaluating the GPSR which is very popular Position Based Routing protocol. In Location based approaches with the help of on-board navigation systems, the vehicles are aware of their geographical positions.

E. Fonseca and A. Festag [7]did a survey on existing approaches for secure ad-hoc routing. They found that the major attacks in VANETs are impersonation, message forging, black hole, packet dropping, grey hole, worm hole, on-board tampering, and in-transit traffic tampering.

They concluded that VANET uses infrastructure for handling the security by providing private keys to vehicles at Real time. These assigned keys can work well but they need full infrastructure support. Storing keys in the vehicles can also not be a solution as it is totally open to attackers of the network.

C. Harsch, A. Festag, and P. Papadimitratos [8]did a survey on secure position-based routing for VANETs. They proposed defense mechanisms, relying both on cryptographic scheme 
and plausibility checks technique. In this paper to achieve security in VANETs, cryptographic scheme is used and they concluded that their implementation and initial measurements show the proposed scheme deployable and security overhead is low. Plausibility checks reduce the impact of false positions on the routing operation.

N.W. Lo and H.C. Tsai [9]did a survey on Illusion attack on VANET applications i.e. message plausibility problem. In this paper, they found a new attack that is specific to VANETs, called Illusion attack is described and a possible solution to address this attack is proposed through a plausibility mechanism.

In this paper they have presented the illusion attack, a severe security threat that can manipulate a driver's behavior in vehicular ad hoc networks. Also they concluded that in the illusion attack, a malicious attacker creates specific traffic situation and sends the fraud traffic warning messages to decoy other drivers to believe there is a traffic event occurred.

M. Raya and J. P. Hubaux [10]did a survey on securing vehicular ad-hoc networks. They proposed a secure architecture; the architecture consists of the certification authority (CA) where each authority is responsible for a region. Each authority provides certificates to nodes registered with it as well as foreigner certificates to nodes registered with other CAs when these nodes enter its geographical boundary.

They have concluded why vehicular networks need to be secure. They also proposed a model that identifies the most relevant communication aspects; they also identified the major threats. Then they proposed security architecture along with the related protocols; they have shown how and to what extent it protects privacy. They also analyzed the robustness of their proposal.

\section{PERFORMANCE PARAMETERS}

There are some parameters to measure the performance of the GPSR (Greedy Perimeter Stateless Routing) which is very popular in the Position Based Routing. These parameters are used to evaluate the performance of the system:

\subsection{Network Routing Load (NRL)}

It is used to balance the traffic without the use of complex routing across the two WAN links. When forwarding a packet, it allows the router to use multiple paths to send the packet to its destination and this must be decrease the improvement in the network performance.

\subsection{End to end delay (E2E delay)}

End to end delay is the time taken by a packet to be transmitted through a network from source to destination. Also end to end delay is the delay of the each node to the total packets transmitted through the network.

\subsection{Packet Delivery Ratio (PDR)}

Basically the packet delivery ratio is the ratio of the packets delivered successfully to the destination as compared to the number of packets transferred by the sender. To improve the network performance this PDR must be maximum.

\subsection{Network Throughput}

Throughput of the network is defined as the total number of packets received per unit time at the server. Throughput is measured in bits/sec and for the improvement in the network performance this must be increased.

\section{SIMULATION ENVIRONMENT}

In the present study, it is clearly shown that the inaccuracy occurred in Position Based Routing is due to the two types of mobility parameters i.e. speed of moving nodes and the packet interval time. There are some parameters like Throughput, Average End to End delay, Network Routing Load and Packet Delivery Ratio are calculated to measure network performance for GPSR (Greedy Perimeter Stateless Routing) which is popular in Position Based Routing.

\section{Simulation in SUMO and NS2}

In this paper we are considering the Delhi map which is shown in the fig 1 . In the SUMO the information of the network topology and route files i.e. net.xml and rou.xml respectively are calculated by using Net converter and Dua router. In the real time scenario, the communication between the nodes is bi-directional. So that a TCP connection is required for the nodes to behave like a sender or receiver.

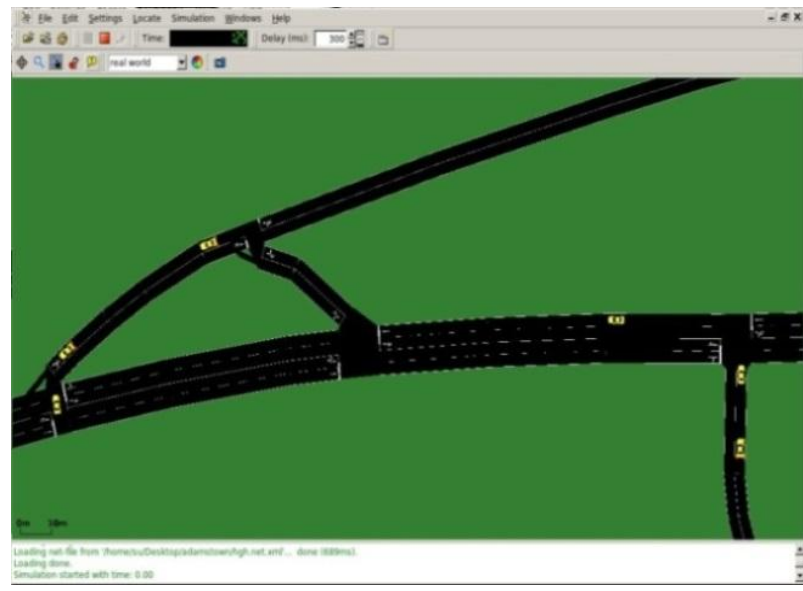

Fig 1: Moving Vehicles in Delhi map shown in SUMO

The mobility of the vehicles is controlled by the SUMO but the nodes are controlled by the Network Simulator 2. It is shown in the fig 2 that a TCP packet is generated and sent by a node and it is received by another (neighbor) node.

In fig 2 it is shown that a packet is send by a node and it is received by another node. Now how the network will knowthe packet is received by the node or not. So anacknowledgement is send by the node who received the TCP packet which is shown in fig 3 .

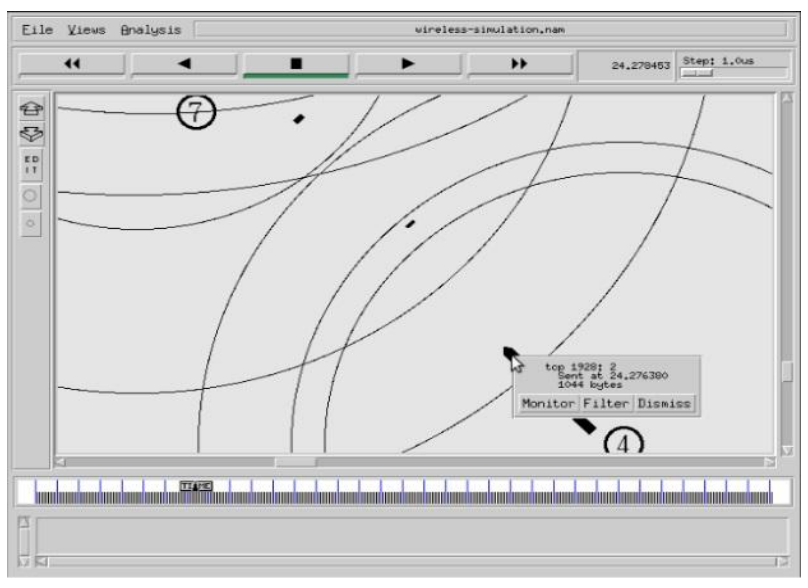

Fig 2: TCP Packet is send by a node in NS2 


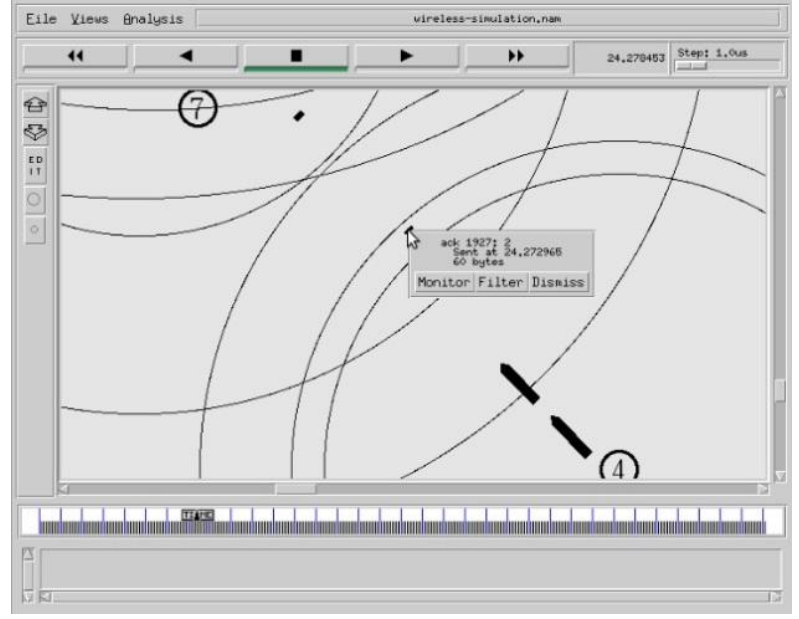

Fig 3: Acknowledgement is send back in NS2

It is clearly shown by the acknowledgement in fig 3 to ensure that the TCP packet is received by the node. Now these are ideal cases, but in some case the TCP packet get dropped and will not receive by the node. This drop of packet is shown in fig 4 given below:

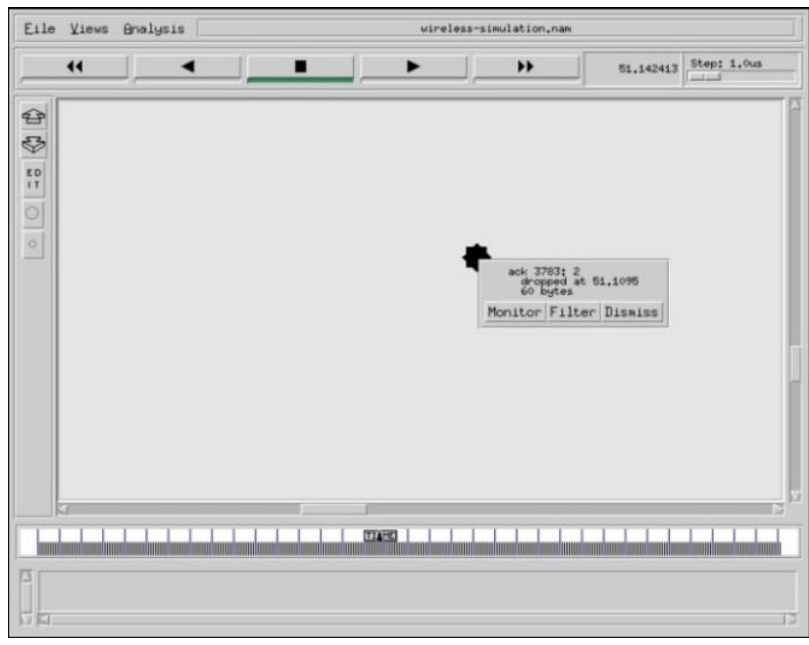

Fig 4: TCP packet is dropped in NS2

These are some figures showing the movement of the nodes in SUMO (Simulation for Urban Mobility) and NS2 (Network Simulator 2). Also the TCP packet is send and received by the nodes. The acknowledgement is also send by the nodes and in some cases the TCP packet is dropped.

\section{RESULTS AND DISCUSSIONS}

The performance for the GPSR which is popular in PBR is evaluated in the form of some parameters i.e. Throughput, End to end delay, Network routing load and the packet delivery ratio.

Throughput of the network is defined as the total number of packets received per unit time at the server. Throughput is measured in bits/sec and for the improvement in the network performance this must be increased.

End to end delay is the time taken by a packet to be transmitted through a network from source to destination. Also end to end delay is the delay of the each node to the total packets transmitted through the network.

Network routing load is used to balance the traffic without the use of complex routing across the two WAN links. When forwarding a packet, it allows the router to use multiple paths to send the packet to its destination and this must be decrease to improve the network performance.

Basically the packet delivery ratio is the ratio of the packets delivered successfully to the destination as compared to the number of packets transferred by the sender. To improve the network performance this PDR must be maximum.

These are some parameters used in this paper to evaluate the performance of the Position based routing, and the results are shown in the form of a graph shown below.

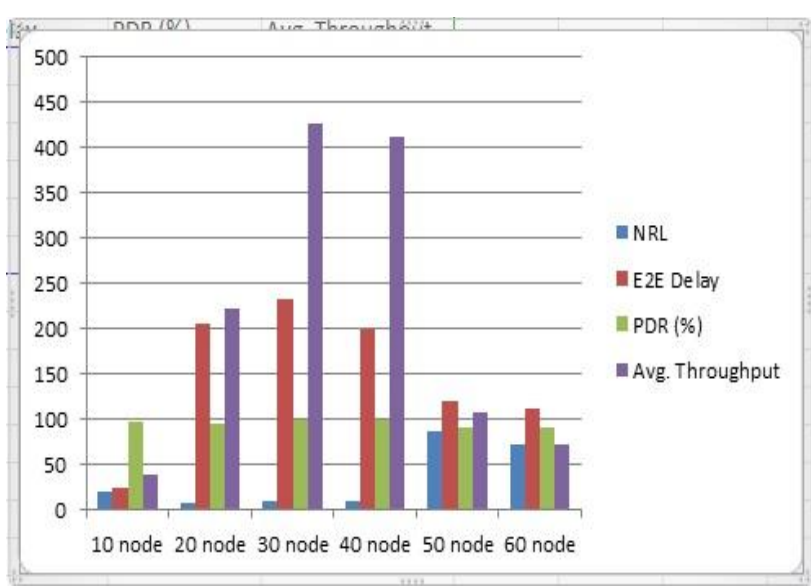

\section{Fig 5: Results of performance parameters shown in the form of graph.}

Due to the path instability and network disconnection the change in the topology occurs, as a result the performance of the PBR decreases with the increase in the speed of nodes. The speed of vehicles generates the inaccuracy in receiving the geographical information of nodes [16].

\section{CONCLUSION AND FUTURE WORK}

Over the last few years lot of research have been done in the field of VANET. In this paper an overview of GPRS which is popular in Position Based Routing is discussed focusing on various performance parameters. Network topology and the route information about the Delhi region (Urban city) is obtained by the use of Open street map. Both the SUMO and NS2 is used to realize a real time scenario. The results are evaluated by the various performance parameters. The values of throughput and the packet delivery ratio must be maximized but the network routing load and end to end delay must be minimized for the increase in the performance of the network. In future we are preparing to work on the security techniques to detect the malicious nodes and also to eliminate the malicious nodes, because the PBR does not handle the false location generation [17]. Also the PBR uses digital signatures which require an additional infrastructure.

\section{REFERENCES}

[1] SheraliZeadalli, Ray Hunt, Yuh-Shyan Chen ,Angela Irwin and Aamir Hassan "Vehicular ad hoc networks (VANETS) status, results and challenges”, Telecommunication Systems, DOI 10.1007/s11235-0109400-5, (C) Springer Science + Business Media, LLC 2010.

[2] Md. HumayunKabir, "Research Issues on Vehicular Ad hoc Network", International Journal of Engineering Trends and Technology (IJETT) - Volume 6, Number 4, pp. 174-179, Dec 2013. 
[3] Yasser Toor And Paul Muhlethaler, InriaAnisLaouiti, Get/Int Arnaud De La Fortelle, Ecole Des Mines, "Vehicles Ad hoc networks: Applications and Related Technical Issues", IEEE Communications Surveys \& Tutorials, 3rd Quarter 2008, Volume 10, No. 3, pp. 7488.

[4] DaxinTian, KavehShafiee and Victor C. M. Leung "Position Based Directional Vehicular Routing",Global Telecommunications Conference, 2009, IEEE pp. 16, DOI: 10.1109/GLOCOM.2009.

[5] Hannes Hartenstein, Dieter Vollmer and HolgerFubler, "Location-Based Routing for Vehicular Ad-hoc Networks", MOBICOM'02, September 23-28, 2002, Atlanta, Georgia, USA.

[6] M. Mauve, J. Widmer and H. Hartstein, "A Survey on Position Based Routing on Mobile Ad-hoc Networks", IEEE Networks, Volume-15, Issue-6, pp. 30-39, NovDec 2001.

[7] E. Fonseca and A. Festag, "A survey of existing approaches for secure ad hoc routing and their applicability to VANETs", NEC Network Laboratories, Heidelberg, Germany, NLE-PR-2006-19, Mar. 2006.

[8] C. Harsch, A. Festag, and P. Papadimitratos, "Secure position-based routing for VANETs", in Proc. Veh. Technol. Conf., 2007, pp. 26-30.

[9] N.W. Lo and H.C. Tsai, "Illusion attack on VANET applications- A message plausibility problem", in Proc. 2nd IEEE Workshop Autom.Network Appl., Nov. 2007, pp. $1-8$.

[10] M. Raya and J. P. Hubaux,"Securing vehicular ad hoc networks", J. Comput. Security, vol.15, no.1, pp.39-68, 2007.
[11] Hamid Menouar, MassimilianoLenardi and FethiFilali,"Movement Prediction-Based Routing (MOPR) Concept for Position-Based Routing in Vehicular Networks", IEEE $66^{\text {th }}$ Vehicular Technology Conference, 2007.

[12] A. E. Abdallah, T. Fevens and J. Opatrny,"Randomized 3D Position-Based Routing Algorithms for Ad-hoc Networks", $3^{\text {rd }}$ Annual International Conference, 2006.

[13] HamdiIdjmayyel, Bilal R. Qazi and Jaafar M. H. Elmirghani,"Position Based Routing Protocol for a City Environment", Fourth International Conference on Next Generation Mobile Applications, Services and Technologies, pp: 174-179, 2010.

[14] Maryam Mohseni, ShabnamVahedi and MajidNaderi,“A New Position-Based Routing Algorithm for the Reduction of Overhead in Ad-hoc Networks", Second International Conference on Systems and networks Communications, 2007.

[15] Miguel Garcia de la Fuenta and HoudaLabiod, "Performance analysis of position-based routing approaches in VANETs", Mobile Wireless Communications networks, pp: 16-20, 2007.

[16] Dharani N. V, Shylaja B. S and Shree Lakshmi, “ Performance Evaluation of GPSR Routing Protocol for VANETs using Bi-directional Coupling", International Journal of Computer Networks (IJCN), Volume-7, Issue1,2015

[17] Sanjay K. Dhurandher, Mohammad S. Obaidat and AmritJaiswal, "Vehicular Security through Reputation and Plausibility Checks", IEEE System Journal, Vol. 8, No. 2, June 2014. 